\title{
Distinct Contributions of High- and Low-Voltage-Activated Calcium Currents to Afterhyperpolarizations in Cholinergic Nucleus Basalis Neurons of the Guinea Pig
}

\author{
Sylvain Williams, Mauro Serafin, Michel Mühlethaler, and Laurent Bernheim \\ Département de Physiologie, Centre Médical Universitaire, 1211 Genève 4, Switzerland
}

The contributions made by low- (LVA) and high-voltageactivated (HVA) calcium currents to afterhyperpolarizations (AHPs) of nucleus basalis (NB) cholinergic neurons were investigated in dissociated cells. Neurons with somata $>25 \mu \mathrm{M}$ were studied because $80 \%$ of them stained positively for choline acetyltransferase and had electrophysiological characteristics identical to those of cholinergic NB neurons previously recorded in basal forebrain slices. Calcium currents of cholinergic NB neurons first were dissected pharmacologically into an amiloride-sensitive LVA and at least five subtypes of HVA currents. Approximately $17 \%$ of the total HVA current was sensitive to nifedipine ( $3 \mu \mathrm{M}), 35 \%$ to $\omega$-conotoxin-GVIA (200-400 $\mathrm{nm}), 10 \%$ to $\omega$-Agatoxin-IVA (100 nM), and $20 \%$ to $\omega$-AgatoxinIVA (300-500 nM), suggesting the presence of L-, N-, P-, and $\mathrm{Q}$-type channels, respectively. A remaining current (R-type) resistant to these antagonists was blocked by cadmium (100-
$200 \mu \mathrm{M})$. We then assessed pharmacologically the role that LVA and HVA currents had in activating the apamin-insensitive AHP elicited by a long train of action potentials (SAHP) and the AHP evoked either by a short burst of action potentials or by a single action potential (mAHP) that is known to be apamin-sensitive. During sAHPs, $\sim 60 \%$ of the hyperpolarization was activated by calcium flowing through N-type channels and $\sim 20 \%$ through P-type channels, whereas T-, L-, and Q-type channels were not involved significantly. In contrast, during mAHPs, N- and T-type channels played key roles ( $\sim 60$ and $30 \%$, respectively), whereas L-, P-, and Q-type channels were not implicated significantly. It is concluded that in cholinergic NB neurons various subtypes of calcium channels can differentially activate the apamin-sensitive mAHP and the apamin-insensitive sAHP.

Key words: acetylcholine; afterhyperpolarization; arousal; basal forebrain; calcium currents; low-threshold spike
Cholinergic neurons of the nucleus basalis (NB) represent the principal source of cholinergic fibers that innervate the cerebral cortex (for review, see Jones, 1993; Mesulam, 1995). Because this area receives numerous inputs from brainstem nuclei (Jones, 1993) and because acetylcholine has been demonstrated to exert an excitatory action on cortical neurons (for review, see McCormick, 1992), the NB is viewed as a major relay of a cortical activating system implicated in the control of arousal (Jones, 1994). Cholinergic neurons of the NB differ from those of the medial septum (Markram and Segal, 1990; Gorelova and Reiner, 1996) by their localization, projections, and electrophysiological properties. They are distinguished in particular by their ability to discharge in rhythmic bursts of action potentials (APs) when they are activated from a hyperpolarized level (Khateb et al., 1992, 1995; Alonso et al., 1996), whereas they fire tonically when they are depolarized from rest. These rhythmic bursts are subtended by strong low-threshold calcium spikes resulting from the presence in these cells of an important low-voltage-activated (LVA) calcium current (Khateb et al., 1992; Allen et al., 1993; Griffith et al., 1994).

Received Jan. 21, 1997; revised July 18, 1997; accepted July 22, 1997.

This work was supported by grants from the Swiss Fonds National to L.B. and M.M. S.W. was supported by a postdoctoral fellowship from the Medical Research Council of Canada. We thank Dr. Nicholas Saccomano of Pfizer Central Research Laboratories for the generous gift of $\omega$-Agatoxin-IVA, Danièle Machard for her technical assistance, Gilbert von Kaenel for his help with graphics, and Drs. Douglas Fraser and Dennis Churchill for critical reading of an earlier version of this manuscript.

Correspondence should be addressed to Dr. M. Mühlethaler, Département de Physiologie, Centre Médical Universitaire, 1 Rue Michel-Servet, 1211 Genève 4, Switzerland.

Copyright (C) 1997 Society for Neuroscience $\quad 0270-6474 / 97 / 177307-09 \$ 05.00 / 0$
The afterhyperpolarization (AHP) that follows a burst of APs (mAHP) in NB cells is apamin-sensitive (Khateb et al., 1995) and as such is similar to the AHP evoked by a single AP in cholinergic basal forebrain neurons (Matthews and Lee, 1991; Gorelova and Reiner, 1996). In contrast, in the same type of cells, long trains of APs elicit a long-lasting AHP (sAHP) that is apamin-insensitive (Matthews and Lee, 1991; Gorelova and Reiner, 1996). These two types of AHPs (sensitive or insensitive to apamin) are both initiated by calcium influx through voltage-gated calcium channels, but the precise contribution of the various calcium current subtypes to the genesis of AHPs is not known in these cells and is, in general, still a matter of debate in central neurons (Sah, 1996).

The major goal of the present study was thus to investigate in dissociated cholinergic NB neurons the involvement of the different subtypes of calcium currents in the activation of these two delayed AHPs. The underlying hypothesis was that different firing patterns (tonic vs bursting) could elicit AHPs that depend on contributions made by different calcium channels. We were interested in particular to determine whether the T-type current could play a role in the mAHP, because this current is a major determinant of the burst pattern of discharge typical of these cells. To our knowledge a contribution of the T-type current to mAHP has never been demonstrated before.

A preliminary goal for understanding the respective contribution of the various calcium current subtypes to the AHPs was to dissect them pharmacologically by using calcium channel antagonists. In previous studies only $\mathrm{T}$-, L-, and N-type calcium currents were described in cholinergic basal forebrain neurons (Allen et al., 1993; Griffith et al., 1994; Williams et al., 1997). 
However, because coapplication of nifedipine and $\omega$-conotoxinGVIA did not block the total high-voltage-activated (HVA) current completely, additional subtypes must be present in these cells. P-, Q-, and R-type HVA currents now have been identified in various preparations (Mintz et al., 1992; Sather et al., 1993; Stea et al., 1994; Randall and Tsien, 1995). Hence, in addition to T-, L-, and N-type currents, these more recently described subtypes also might be present in cholinergic NB neurons and possibly contribute to activate AHPs.

In this report we present evidence that the N-type current plays a key role in activating both apamin-sensitive and apamininsensitive AHPs in cholinergic NB neurons. In addition, depending on the firing pattern, either P- or T-type currents also contribute to the activation of AHPs and thus could participate in regulating the firing properties of these cells.

\section{MATERIALS AND METHODS}

Dissociation. Slices from young guinea pigs $(80-200 \mathrm{gm})$ were obtained by standard methods (Khateb et al., 1992, 1993) and were dissociated with a slightly modified version of the method developed by Kay and Wong (1986). Briefly, guinea pigs were anesthetized deeply with Nembutal and decapitated. The brain was removed and rapidly transferred in cold $\left(4^{\circ} \mathrm{C}\right)$ oxygenated $\left(95 \% \mathrm{O}_{2} / 5 \% \mathrm{CO}_{2}\right)$ physiological saline containing (in mM): $130 \mathrm{NaCl}, 20 \mathrm{NaHCO}_{3}, 1.25 \mathrm{KH}_{2} \mathrm{PO}_{4}, 1.3 \mathrm{MgSO}_{4}, 5 \mathrm{KCl}, 10$ glucose, and $2.4 \mathrm{CaCl}_{2}, \mathrm{pH} 7.35$. With a vibratome (Campden Instruments, Berlin, Germany), two to three slices (400 $\mu \mathrm{M}$ thick) containing the basal forebrain were cut and left at room temperature for a period of $1 \mathrm{hr}$ in physiological saline. For dissociations, slices were immersed first in a $100 \%$ oxygen-equilibrated PIPES solution containing (in mM): 120 $\mathrm{NaCl}, 5 \mathrm{KCl}, 0.5 \mathrm{CaCl}_{2}, 1 \mathrm{MgCl}_{2}, 25$ glucose, and $20 \mathrm{PIPES}, \mathrm{pH}-$ adjusted to 7.0 with $\mathrm{NaOH}$. Then the region of interest, which included the substantia innominata, the horizontal limb of the diagonal band, and the magnocellular preoptic nucleus [Paxinos and Watson (1986); see Gritti et al. (1993) for a discussion on basal forebrain cholinergic nuclei], was dissected out (one piece from each hemisphere) with a small razor blade. The regions of the medial septum and the vertical limb of the diagonal band containing most of the septohippocampal-projecting neurons were excluded. In this study the term NB neurons will be used to characterize those cells located within the region of interest mentioned above. The tissue was placed in small test tubes containing an oxygenated PIPES solution with the enzyme trypsin (Sigma type XII, $0.8 \mathrm{mg} / \mathrm{ml}$; St. Louis, MO) for $120 \mathrm{~min}$ at $30^{\circ} \mathrm{C}$. The enzymatic reaction was stopped by rinsing the tissue with $10 \%$ goat or horse serum (Life Technologies, Gaithersburg, MD) and left to rest in enzyme-free PIPES for at least another hour. Neurons were dispersed by triturating in HEPES (see below) with two different sizes of fire-polished Pasteur pipettes. The suspension was transferred to a Cell-tak (Becton Dickinson Labware, Heidelberg, Germany)-coated Petri dish, which was mounted on an inverted microscope (Zeiss, Oberkochen, Germany). Healthy neurons adhered to the bottom of the dish within $15 \mathrm{~min}$.

Whole-cell recording and solutions. Voltage-clamp recordings from neurons were obtained by using the whole-cell version of the patch-clamp technique (Hamill et al., 1981). Patch pipettes were pulled (Sutter Instruments, Novato, CA) from borosilicate glass (1.5 outer diameter and 0.86 inner diameter; Clark Electromedical Instruments, Pangbourne, UK) and had resistances typically of 2-4 M $\Omega$ in the bath solution. Signals were recorded with an Axopatch 200A amplifier (Axon Instruments, Foster City, CA) and monitored on a 486 PC clone equipped with pClamp software (version 6.0) and a $125 \mathrm{kHz}$ interface (Digidata 1200 , Axon Instruments). Records were low-pass-filtered at 2 or $5 \mathrm{kHz}$ with a Bessel filter. Typical access resistances ranged from 4-8 $\mathrm{M} \Omega$, and compensation of $70-80 \%$ was used. Linear leak current and capacitive transients were subtracted by using a $\mathrm{P} / 4$ protocol or by subtracting raw traces from those in the presence of cadmium $(100-200 \mu \mathrm{M})$. The internal recording solution contained the following (in $\mathrm{mm}$ ): $130 \mathrm{Cs}-$ methylsulfonate, $20 \mathrm{CsCl}, 5 \mathrm{MgCl}_{2}$, 5 HEPES, $3 \mathrm{Na}_{2} \mathrm{ATP}, 0.2 \mathrm{GTP}-\mathrm{Na}$, 0.1 BAPTA, and 14 phosphocreatine, $\mathrm{pH}$-adjusted to 7.3 with $\mathrm{CsOH}$. Neurons were perfused continuously $(3 \mathrm{ml} / \mathrm{min})$ with a HEPES medium containing (in $\mathrm{mM}$ ): $150 \mathrm{NaCl}, 2.5 \mathrm{KCl}, 2 \mathrm{CaCl}_{2}, 1 \mathrm{MgCl}_{2}, 8$ glucose, and 10 HEPES, pH-adjusted to 7.3 with $\mathrm{NaOH}$. Calcium currents were isolated by adding tetrodotoxin ( $1 \mu \mathrm{M}$, Latoxan), tetraethylammonium $(20 \mathrm{~mm})$, and 4-aminopyridine (4 mM) to the HEPES medium. To study membrane properties and AHPs in current-clamp mode, we perfused isolated neurons with a HEPES medium (see above) and recorded them with an internal solution containing (in $\mathrm{mM}$ ): $121 \mathrm{~K}$-methylsulfate (Pfaltz \& Bauer, Waterbury, CT), $4.5 \mathrm{KCl}, 5 \mathrm{MgCl}_{2}, 5 \mathrm{HEPES}, 3 \mathrm{Na}_{2} \mathrm{ATP}, 0.1$ GTP, 0.1 BAPTA, and 14 phosphocreatine, $\mathrm{pH}$-adjusted to 7.4 with $\mathrm{NaOH}$.

Drugs were applied close to the recorded neuron by a multibarreled pipe system constructed with polyethylene tubing according to the method of Bertrand et al. (1997). All drugs were prepared in single-use aliquots and thawed on the day of the experiment. Amiloride (Sigma), $\omega$-conotoxin-GVIA (Alomone Labs, Jerusalem, Israel), and $\omega$-AgatoxinIVA (gift from Pfizer, New York, NY) were prepared in distilled water and kept frozen at $-20^{\circ} \mathrm{C}$ until the day of the experiment. Agatoxin-IVA was aliquoted with cytochrome $c(0.05 \%$, Sigma) to prevent nonspecific binding to plastic tubing (Mintz et al., 1992). Nifedipine (Sigma) was diluted in dimethylsulfoxide $(<0.1 \%)$. All experiments were performed at room temperature $\left(20-22^{\circ} \mathrm{C}\right)$.

Run-down of calcium currents was greatly minimized by using Csmethylsulfonate (Zhang et al., 1994) supplemented with ATP and phosphocreatine in the patch pipette (McDonough et al., 1996), by using test pulses that were only $20-40 \mathrm{msec}$ in duration (Murchison and Griffith, 1996), and, finally, by activating currents no more than once every 7 sec. In these conditions, run-down of the HVA calcium current was estimated to be $3.7 \%$ per $3 \mathrm{~min}(n=3)$. Access resistance was checked periodically. The amplitude of the LVA and HVA currents was measured at the peak. The amplitude of the AHP after a burst of APs was determined by taking the difference between a point $50 \mathrm{msec}$ before the beginning of the first AP and the value at the peak of the AHP. For the AHP evoked by a train of depolarizing current steps (each one eliciting a single AP), measurement of the amplitude was taken at the peak of the AHP from the average of two or three traces. All means are expressed as mean \pm SE.

Immunocytochemistry. The tissue containing the NB was treated with trypsin as described above. Then the tissue was triturated in PBS, $\mathrm{pH} 7.4$, and neurons were dispersed onto a Cell-tak-coated Petri dish. Neurons were rinsed for $45 \mathrm{sec}$ in PBS containing $4 \%$ paraformaldehyde (Fluka, Neu-Ulm, Germany) and for $5 \mathrm{~min}$ in PBS containing $0.02 \%$ Triton X-100 (Sigma). Afterward, neurons were incubated for $20 \mathrm{~min}$ with a primary rat antibody for choline acetyltransferase (ChAT) Khateb et al., 1992) (dilution 1:250; Jackson ImmunoResearch, West Grove, PA). Nonspecific immunostaining was reduced by rinsing neurons for 20 min with PBS containing $10 \%$ normal goat serum. Then the neurons were incubated with a secondary sheep FITC-conjugated anti-rat antibody (Sig$\mathrm{ma}$ ) for $20 \mathrm{~min}$ (dilution 1:320) and finally were rinsed in PBS for another $20 \mathrm{~min}$. The size of the neurons was estimated by measuring the longer axis of their soma, excluding the proximal portion of primary dendrites.

\section{RESULTS}

\section{Identification of neurons}

Neurons in the NB stain positively for either glutamic acid decarboxylase (GAD), the synthesizing enzyme for GABA, or for ChAT, the synthesizing enzyme for acetylcholine (Gritti et al., 1993). It has been suggested from histological analysis that these two populations have similar morphologies (both being bior multipolar) but may be distinct with respect to the size of the soma (Nakajima et al., 1985; Gritti et al., 1993). Hence, ChAT immunostaining was applied to dissociated NB neurons to characterize the size of the soma of both ChAT-positive and ChATnegative cells (Fig. 1A). Neurons that stained positively for ChAT were generally larger in size than those that did not, because $80.9 \%(n=93)$ of neurons $>25 \mu \mathrm{M}$ were ChAT-positive. In contrast, neurons $<25 \mu \mathrm{M}$ comprised a mixed population of neurons. For example, $47.0 \%(n=39)$ of cells that had a soma between 16 and $25 \mu \mathrm{M}$ were found to be ChAT-positive. Therefore, to maximize the probability of recordings from neurons with a cholinergic phenotype, we sampled only neurons $>25 \mu \mathrm{M}(29 \pm$ $3 \mu \mathrm{m}, n=61)$ in this study.

Cholinergic NB neurons have been shown in basal forebrain slices to display electrophysiological characteristics distinct from those of noncholinergic neurons (Khateb et al., 1992; Alonso et al., 1994, 1996). Indeed, cholinergic NB neurons show single- 
A

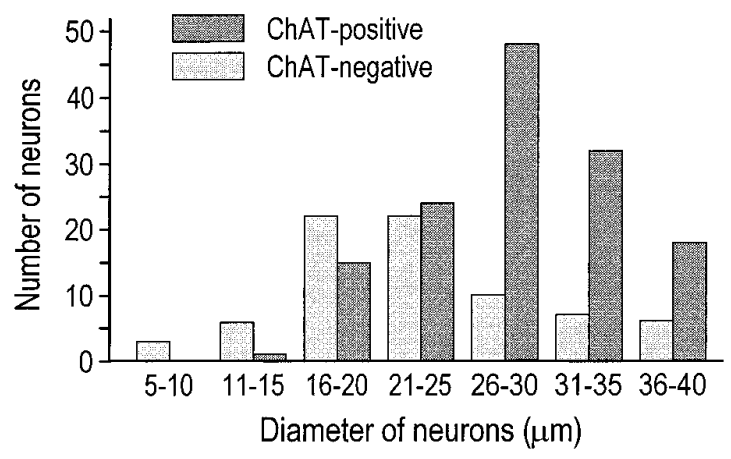

C

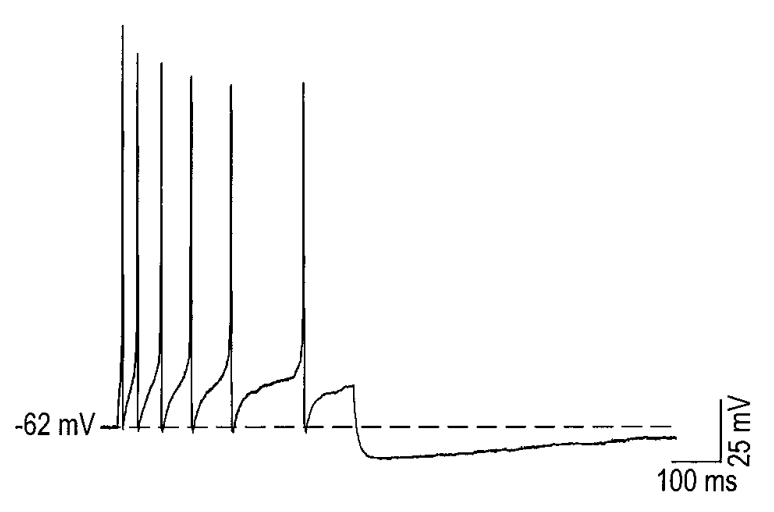

B

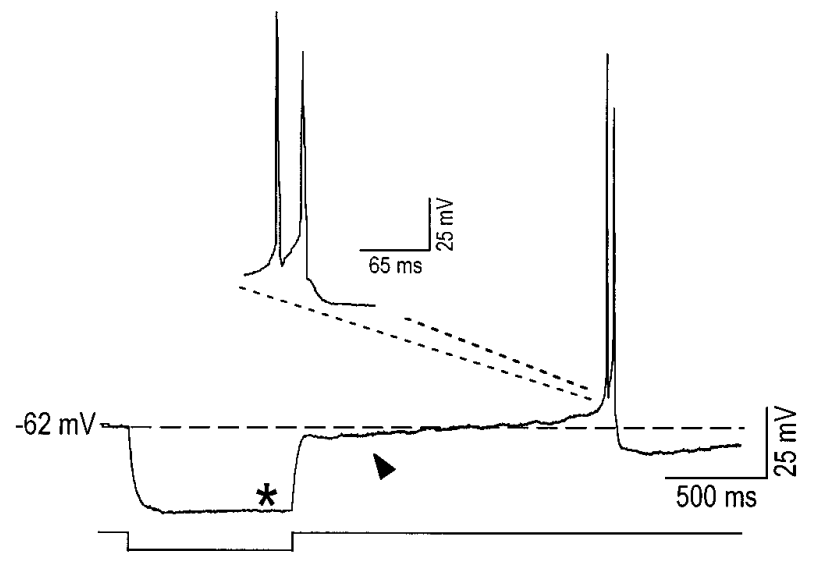

D

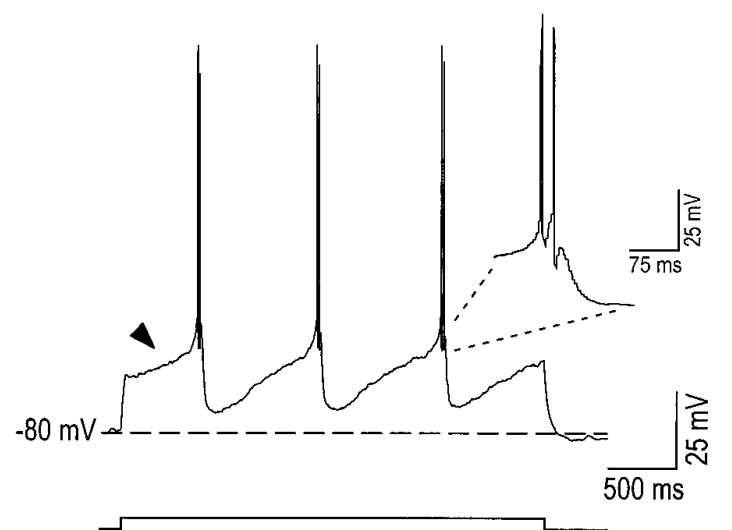

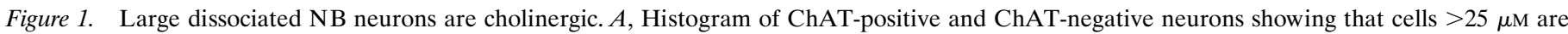

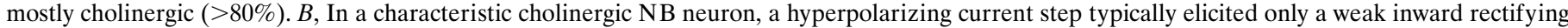

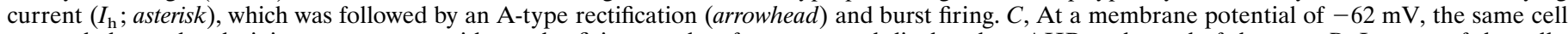

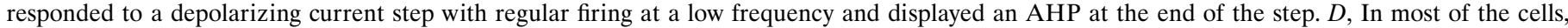

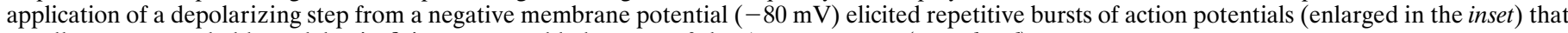
usually were preceded by a delay in firing, presumably because of the A-type current (arrowhead).

spike low-frequency tonic firing when activated near rest, but they display calcium-dependent low-threshold spikes (LTS) that can trigger multiple APs when they are activated from a hyperpolarized level. In addition, they exhibit a transient rectification (A-like current) that precedes the LTS and seldom display an inward rectification (Khateb et al., 1992; Alonso et al., 1996). In the present study, dissociated NB neurons $>25 \mu \mathrm{M}$ that were recorded with patch electrodes displayed the same electrophysiological properties. Results presented in Figure 1 show that these cells (57/61) displayed an LTS when they were depolarized from a membrane potential more negative than rest (Fig. $1 D$ ), but they responded, in contrast, with a slow tonic firing when they were depolarized from rest (Fig. $1 C$ ). In addition, whereas they generally displayed a prominent A-like current (56 of 61; arrowhead in Fig. $1 B, D)$, they demonstrated an inward rectification only rarely (4 of 52; asterisk in Fig. $1 B$ ). Thus, the electrophysiological properties and the ChAT immunostaining both indicate that neurons with a soma $>25 \mu \mathrm{M}$ are mostly cholinergic. In the present study NB cholinergic neurons had a resting membrane potential more negative than $-60 \mathrm{mV}$, and, at a depolarized membrane potential of $-52 \pm 4 \mathrm{mV}(n=57)$, APs evoked by small 1-msec-long depolarizing current steps measured $113 \pm 18 \mathrm{mV}$ (from baseline), had a half-width of $1.5 \pm 0.4 \mathrm{msec}$ (at $50 \%$ amplitude), and had a decay time of $1.7 \pm 0.6 \mathrm{msec}$ (from $90 \%$ of peak to $10 \%$ ).

\section{Pharmacology of calcium currents}

HVA calcium currents can be separated into distinct components by using pharmacological antagonists (Mintz et al., 1992; Randall and Tsien, 1995; McDonough et al., 1996). In cholinergic basal forebrain neurons, $\mathrm{L}$ - and $\mathrm{N}$-type calcium currents have been identified in a previous study by using nifedipine and $\omega$-conotoxin-GVIA (cono-GVIA), respectively, but additional subtypes are present because coapplication of these blockers did not inhibit all of the calcium current (Allen et al., 1993). The effects of these L- and $\mathrm{N}$-type channel blockers were confirmed in the present study, and the additional presence of P-and Q-type channels was tested with two different concentrations of $\omega$-Agatoxin-IVA (Aga-IVA). Indeed, although P-type channels are known to be inhibited by 100 nм Aga-IVA (Mintz et al., 1992; Mintz and Bean, 1993; McDonough et al., 1996), larger concentrations $(\geq 200 \mathrm{nM}$ ) are necessary to block Q-type channels (Randall and Tsien, 1995; Ciranna et al., 1996). Hence to assess for the presence of Q-type channels, we always added 300-500 nM Aga-IVA after the addition of $100 \mathrm{~nm}$ Aga-IVA.

The effects of cumulative application of antagonists on the peak calcium current from one experiment are plotted as a function of time in Figure $2 A$, and current traces are displayed in Figure $2 B_{1}$. Results for all neurons with the same experimental 

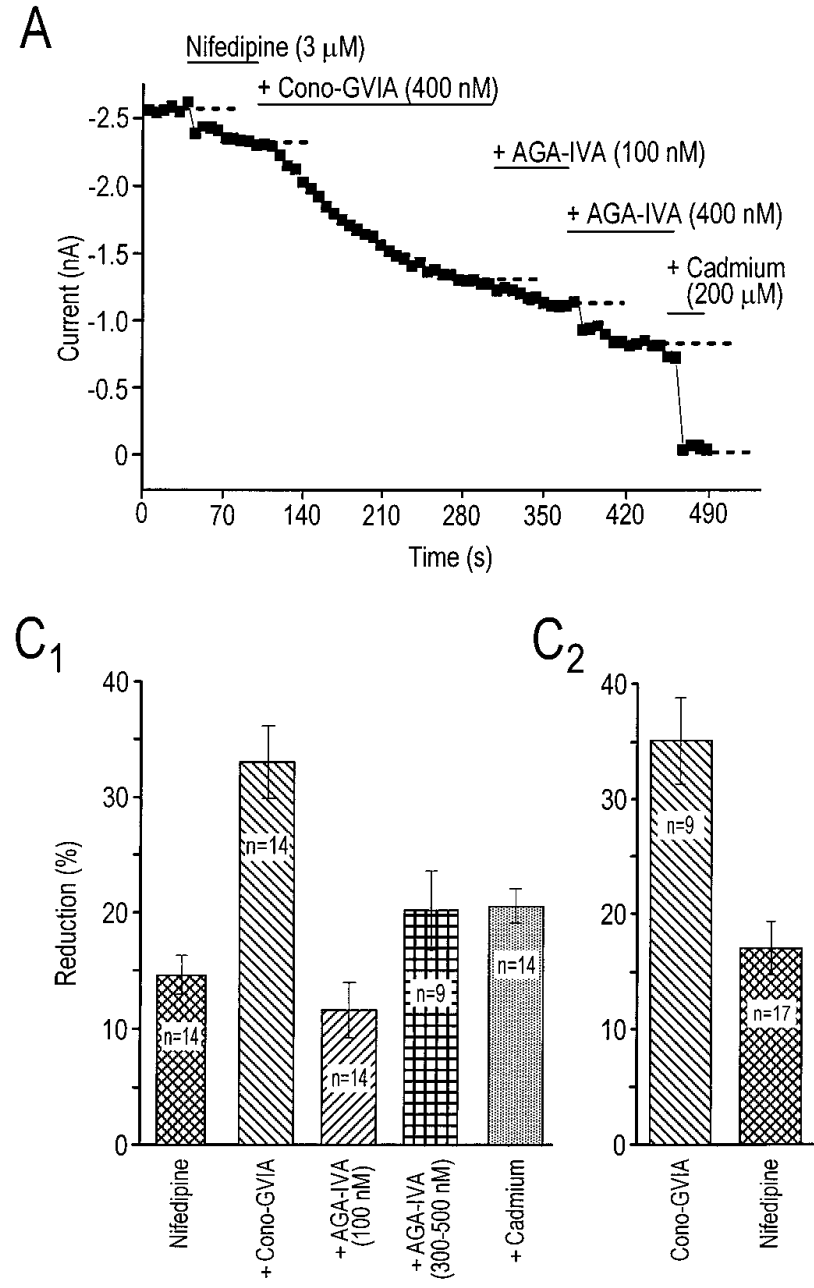

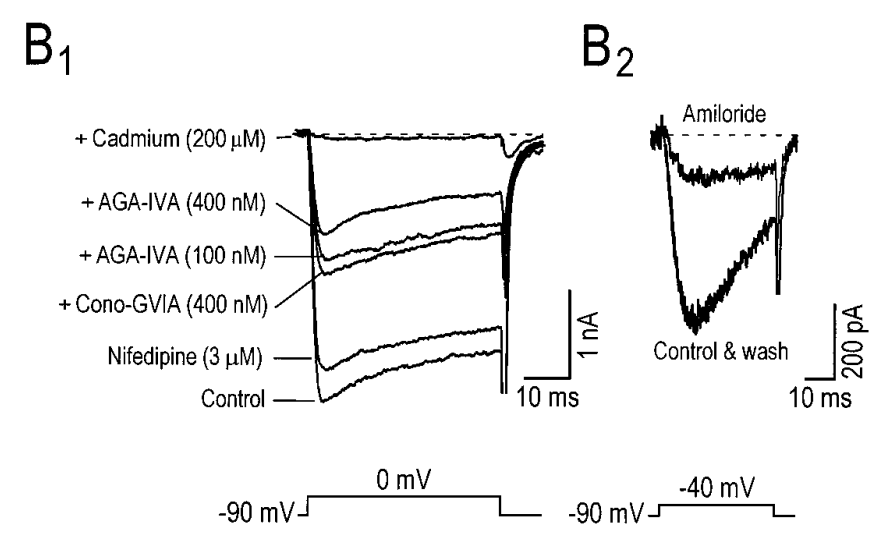

D

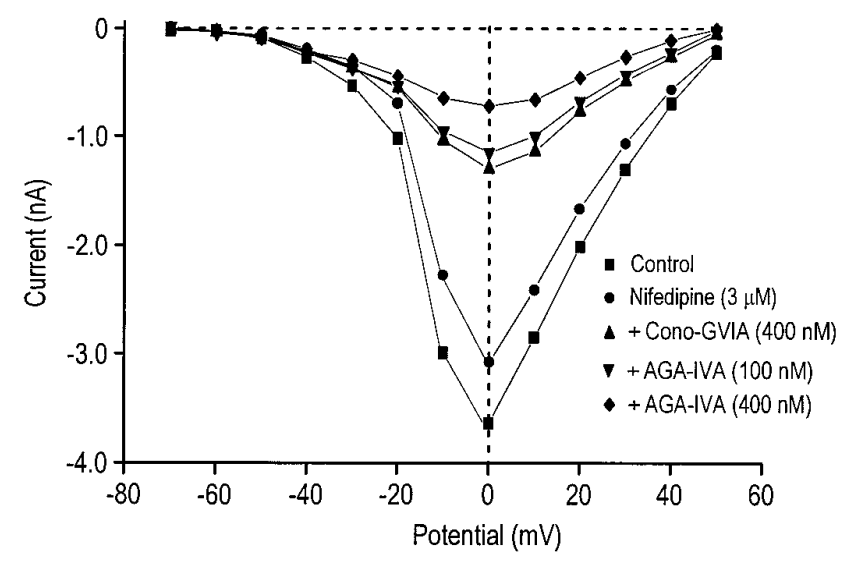

Figure 2. Pharmacological characterization of calcium currents. $A$, Plot of the peak calcium current versus time during cumulative applications of $3 \mu \mathrm{M}$ nifedipine, $400 \mathrm{~nm}$ cono-GVIA, $100 \mathrm{~nm}$ Aga-IV, $400 \mathrm{~nm}$ Aga-IVA, and $200 \mu \mathrm{m}$ cadmium. $B_{1}$, Whole-cell leak-subtracted current traces (same experiment as in $A$ ) elicited by a $40 \mathrm{msec}$ depolarizing step to $0 \mathrm{mV}$ from a $V_{\mathrm{h}}$ of $-90 \mathrm{mV}$. In this cell $12 \%$ of the total HVA calcium current was blocked by nifedipine, $37 \%$ by cono-GVIA, $7 \%$ by Aga-IVA (100 nM), and $12 \%$ by Aga-IVA (400 nM), whereas the remaining current ( $33 \%)$ was eliminated by cadmium $(200 \mu \mathrm{M}) . B_{2}$, Whole-cell leak-subtracted current traces obtained with a depolarizing test pulse to $-40 \mathrm{mV}$ from a membrane potential of -90 $\mathrm{mV}$ (to activate the T-type current) showing the reversible block produced by amiloride $(300 \mu \mathrm{M})$. $C$, Summary of experiments performed with calcium current antagonists, expressed in percentage of reduction over the control, for cumulative $\left(C_{1}\right)$ and separate $\left(C_{2}\right)$ applications. $D$, Peak calcium current as a function of test potential in control conditions and during cumulative applications of calcium antagonists. A LVA current was evident at potentials greater than $-60 \mathrm{mV}$ and was followed by currents that peaked at $0 \mathrm{mV}$.

protocol are shown in Figure $2 C_{1}$. These experiments reveal that $3 \mu \mathrm{M}$ nifedipine blocked $14.6 \pm 1.7 \%, 200-400 \mathrm{~nm}$ cono-GVIA blocked 33.0 $\pm 3.2 \%, 100 \mathrm{~nm}$ Aga-IVA inhibited $11.6 \pm 2.3 \%$, and 300-500 nM Aga-IVA blocked $20.2 \pm 3.4 \%$ of the total calcium current. The effects of separate applications of each antagonist on the whole-cell calcium current were tested also. Figure $2 C_{2}$ shows that $35.2 \pm 3.7 \%$ of the current was blocked by $200-400 \mathrm{~nm}$ cono-GVIA, $17.0 \pm 2.3 \%$ by $3 \mu \mathrm{M}$ nifedipine, and $10.0 \pm 1.8 \%$ by $100 \mathrm{~nm}$ Aga-IVA. Comparison of the extent of inhibition obtained with cumulative antagonist applications to separate antagonist applications revealed that nifedipine did not interfere with the inhibitory effect of cono-GVIA and that coapplication of cono-GVIA with nifedipine did not occlude the effects of AgaIVA (compare Fig. $2 C_{1}, C_{2}$ ). This suggests that these antagonists were specific for their respective HVA channel subtype. To differentiate further those currents sensitive to low and high concentrations of Aga-IVA, we measured and compared their time to peak and decay rates in the same cells in the presence of nifedipine $(3 \mu \mathrm{M})$ and cono-GVIA (400 nM). It was found that, although the decay rate of the currents did not differ between the two groups when 40 -msec-long steps $(n=4)$ were used, the time to peak of the current sensitive to $100 \mathrm{~nm}$ Aga-IVA was twice as fast as that found for the current sensitive to $400 \mathrm{~nm}$ Aga-IVA ( $7.9 \pm 0.4$ vs $4.1 \pm 0.4 \mathrm{msec}, n=4$; data not shown). These results further support the idea that low and high concentrations of Aga-IVA reduce distinct components of the HVA calcium current in cholinergic NB neurons, thus indicating the presence of $\mathrm{P}$ and Q-type currents (Sather et al., 1993; Randall and Tsien, 1995) in these cells. A cadmium-sensitive current was left after the addition of all the antagonists. The threshold of activation of this current also was assessed to determine possible similarities with a previously described R-type (remaining) current (Ellinor et al., 1993) that is insensitive to all HVA blockers and is known to have an activation threshold intermediate between LVA and HVA currents. Figure $2 D$ shows that after the addition of all of the antagonists ( filled symbols), both the threshold of activation and 
the peak current of the remaining component appeared unchanged, peaking at $0 \mathrm{mV}$. Similar results were obtained in three other cells, suggesting that the remaining current in cholinergic NB neurons displays kinetics that are different from those of the previously described R-type current. Despite these differences the " $\mathrm{R}$ " terminology was maintained in the present study because it also described a current resistant to nifedipine, cono-GVIA, and Aga-IVA. Taken together, these results suggest that cholinergic NB neurons possess at least five distinct types of HVA calcium channels: L-, N-, P-, Q-, and R-types.

We also investigated the effects of amiloride and HVA blockers on the LVA calcium current (evoked by a test pulse to $-40 \mathrm{mV}$ from a holding potential of $-90 \mathrm{mV}$ ). Figure $2 B_{2}$ shows that amiloride suppressed the LVA current, as was suggested previously (Tang et al., 1988; Allen et al., 1993). In this cell amiloride reversibly reduced the LVA current by $64 \%$, whereas the HVA current (evoked by a test pulse to $0 \mathrm{mV}$ ) was reduced by only $3 \%$ (data not shown). In five cells, $300 \mu \mathrm{M}$ amiloride reduced the LVA current by $70.8 \pm 1.1 \%$ and the HVA current by only $3.0 \pm 0.8 \%$. Hence, amiloride shows a high blocking specificity for LVA T-type channels in cholinergic NB neurons. Using separate applications of the HVA blockers, we observed that cono-GVIA (300 nM; $n=3$ ) had no effect on the LVA current and Aga-IVA $(100-400 \mathrm{~nm})$ produced a $9.7 \pm 1.8 \%(n=4)$ reduction of this current, whereas $3 \mu \mathrm{M}$ nifedipine significantly reduced it by $32.7 \pm$ $4.2 \%(n=3)$. Therefore, whereas cono-GVIA and Aga-IVA were both relatively specific antagonists of different components of the HVA current, nifedipine also significantly reduced the T-type LVA current [for review, see Akaike (1991); for cholinergic NB neurons, see Allen et al. (1993)].

\section{Calcium currents and the AHP after a train of APs (sAHP)}

Calcium entry through voltage-gated calcium channels is known to be involved in the activation of the AHPs that follow either a single AP or a train of APs in neurons of the NB (Tatsumi and Katayama, 1993). We (Khateb et al., 1995) and others (Matthews and Lee, 1991; Gorelova and Reiner, 1996) have shown that the AHP elicited by either a single AP or by bursts of APs in cholinergic basal forebrain neurons is blocked completely by apamin. On the other hand, the AHP elicited by a train of APs seems to be of a different kind because it is totally apamininsensitive (Matthews and Lee, 1991; Gorelova and Reiner, 1996). In agreement with these studies, we found that $100 \mathrm{nM}$ apamin did not affect the SAHP ( $n=4$; data not shown).

We first assessed the role that each calcium current type played in the apamin-insensitive sAHP. To ensure that the same number of spikes was elicited in the presence of the various calcium channel antagonists, we applied trains of short 1-msec-long depolarizations (each event eliciting one AP). The number (10-20 events) and frequency $(25-100 \mathrm{~Hz})$ of APs within trains were optimized to always elicit AHPs of maximum amplitude. The applications of either $300 \mathrm{~nm}$ cono-GVIA or $100 \mathrm{~nm}$ Aga-IVA led to a decrease in the peak AHP amplitude (arrow in Fig. 3A,B); it is, however, noteworthy that the effect of cono-GVIA was clearly more important. In contrast, $300 \mu \mathrm{M}$ amiloride had no effect on the AHP (Fig. $3 C$ ). The results of separate antagonists applications on the SAHP are summarized in Figure $3 D$. The histogram shows that $59.7 \pm 8.3 \%$ of the sAHP was reduced by cono-GVIA (200-400 nM) and $21.8 \pm 1.5 \%$ by Aga-IVA (100 nM). On the other hand, nifedipine $(3 \mu \mathrm{M})$ reduced the sAHP only by $10.5 \pm$ $2.3 \%$, and both the Q-type channel blocker Aga-IVA at 300-500

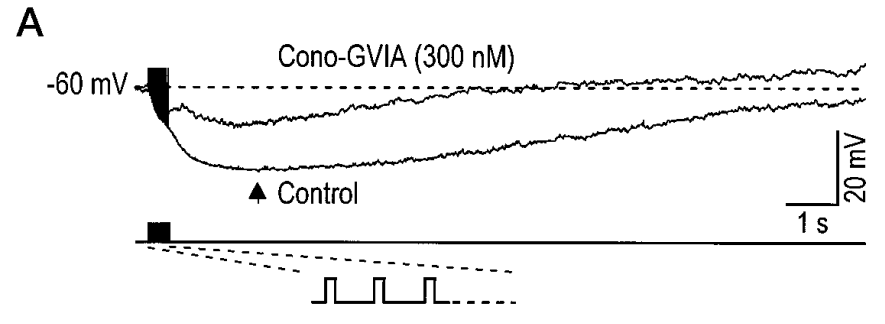

B

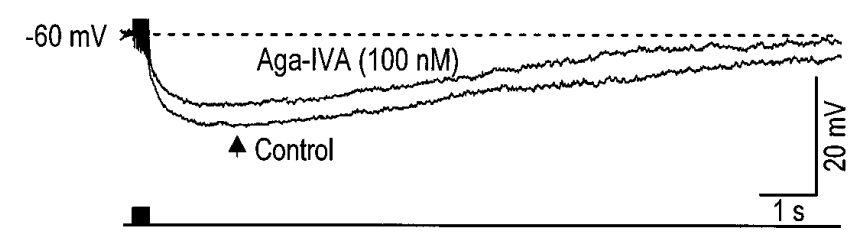

C

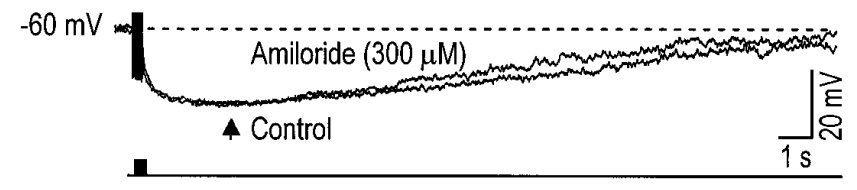

D

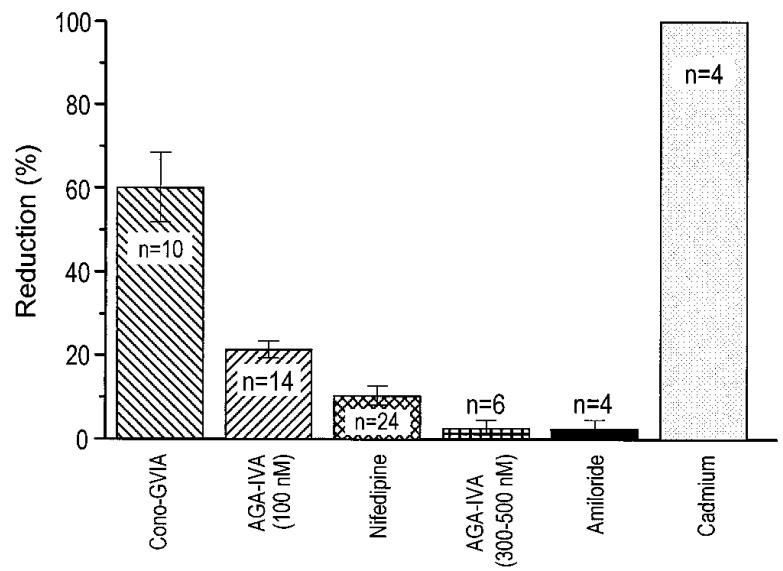

Figure 3. N- and P-type calcium channels are involved in the sAHP. $A$, An AHP evoked by a $400 \mathrm{msec}$ train of 18 depolarizations $(1 \mathrm{msec}$ each in duration) was greatly reduced (measured at arrow indicating the peak amplitude) by the application of $300 \mathrm{~nm}$ cono-GVIA. $B$, In another cell the application of Aga-IVA (100 nM) produced a reduction of $20 \%$ of the sAHP. $C$, The T-type calcium current blocker amiloride $(300 \mu \mathrm{M})$ did not affect the sAHP. $D$, Histogram summarizing the percentage of reduction of the sAHP measured at the peak for separate drug applications. Spike height was truncated in $A-C$.

nм (added after the addition of $100 \mathrm{~nm}$ Aga-IVA) and the T-type channel blocker amiloride $(300 \mu \mathrm{M})$ had only small inhibitory effects on the SAHP. These results suggest that N-type channels play a dominant role in activating the apamin-insensitive sAHP, whereas P-type channels are also involved but to a lesser degree. A significant observation is that the extent of the inhibition by cono-GVIA was similar even if it was applied (in cumulative experiments) after either nifedipine alone $(55.0 \pm 7.7 \%, n=4)$ or nifedipine and Aga-IVA together $(59.0 \pm 3.9 \%, n=3)$. Therefore, the intracellular calcium originating through $\mathrm{N}$-type channels that activated the SAHP could not be substituted with that entering through L- or P-type channels. It should be mentioned 
A

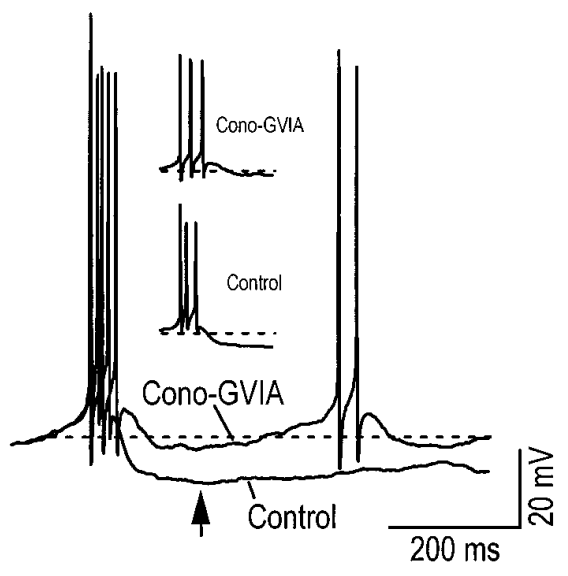

C

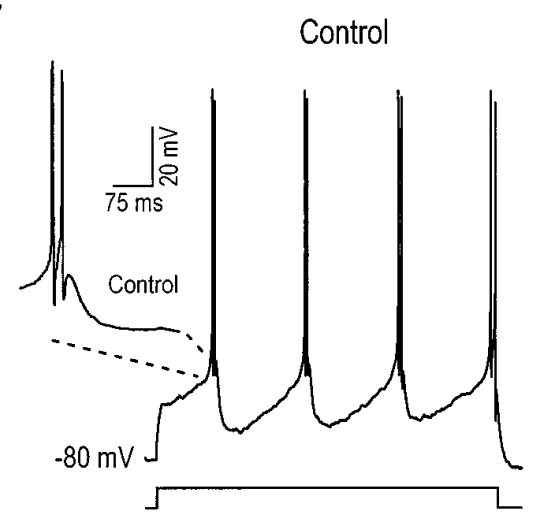

Figure 4. N- and T-type calcium channels are involved in the mAHP. $A$, An mAHP (peak amplitude indicated by arrow) was elicited by a burst of three APs from a membrane potential of -80 $\mathrm{mV}$. Application of $300 \mathrm{~nm}$ cono-GVIA reduced the AHP by $\sim 64 \%$. Note in inset that the MAHP was elicited by the same number of APs in control and in the presence of the antagonist conoGVIA. $B$, Summary of results obtained for separate applications of calcium channel antagonists (cono-GVIA, 200$400 \mathrm{~nm}$; nifedipine, $3 \mu \mathrm{M})$. $C$, $D$, Amiloride $(300 \mu \mathrm{M})$ reduced the amplitude of the mAHP after a burst but also reduced the number of APs in the burst to a single AP. E, In mAHP activated by a single AP (1 msec depolarizing pulse from a potential of $-60 \mathrm{mV}), 300 \mu \mathrm{M}$ amiloride blocked both the DAP (open arrowhead) and a portion of the AHP (arrow), $3 \mu \mathrm{M}$ nifedipine had no effect (data not shown), and $400 \mathrm{~nm}$ conoGVIA (added with both amiloride and nifedipine) blocked the remaining mAHP (cadmium did not reduce the AHP further). $F$, Summary of experiments on mAHP elicited after a single AP. Both amiloride and cono-GVIA significantly reduced the mAHP, whereas $3 \mu \mathrm{M}$ nifedipine (added after amiloride) had no effect on the mAHP.

$E$

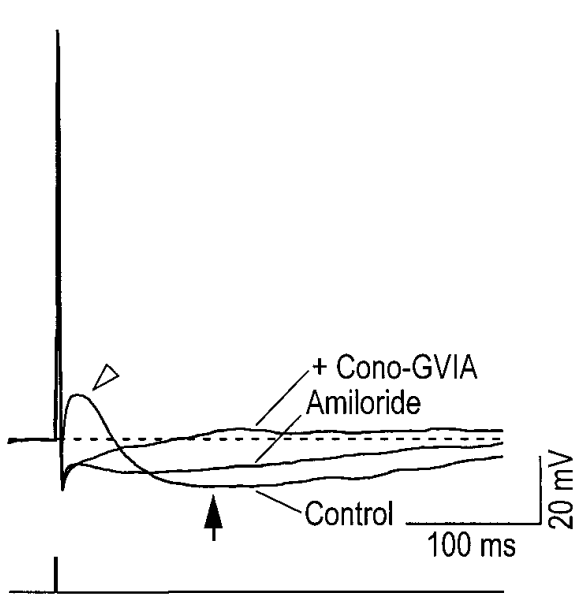

B

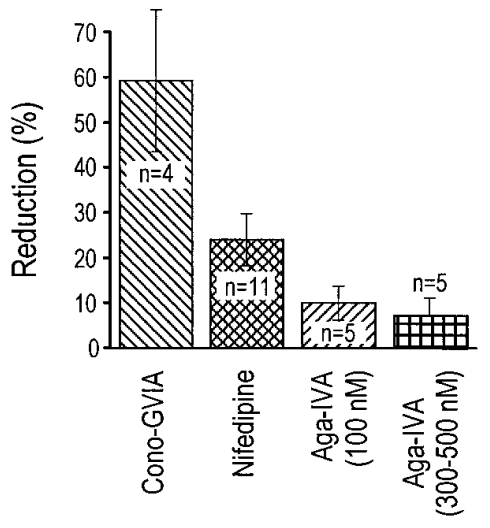

D

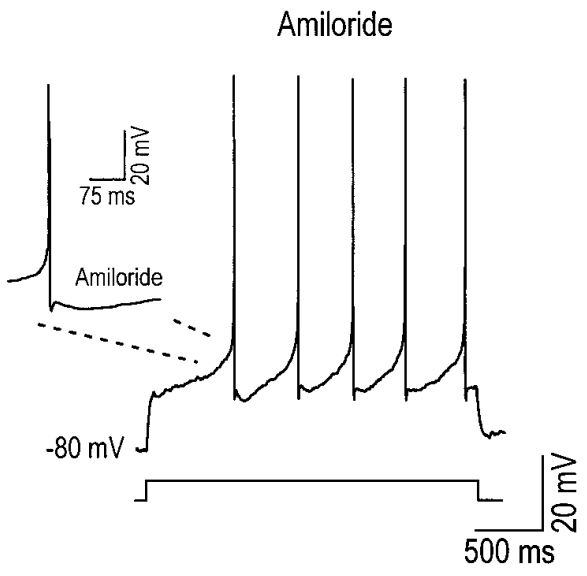

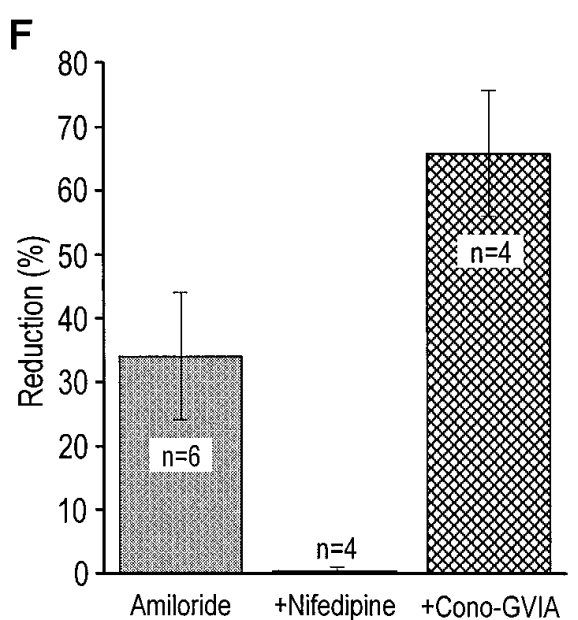

also that a small portion of the SAHP was resistant to all antagonists assayed, but the origin of the calcium could not be determined. This resistant component, eliminated by $100 \mu \mathrm{M}$ cadmium, likely would depend on calcium entry through R-type channels and probably not from ryanodine-sensitive calcium stores, because previous experiments in the $\mathrm{BF}$ have shown that cytoplasmic calcium levels during prolonged membrane depolarization were not altered by ryanodine (Tatsumi and Katayama, 1993).

\section{Calcium currents and the mAHP either after bursts of APs or after single APs}

The respective contribution of LVA and HVA calcium currents in activating the apamin-sensitive mAHP after a burst of APs (Khateb et al., 1995) was assessed also. Bursts (two to three APs) were elicited by depolarizing current steps from a hyperpolarized level of membrane potential $(-80 \mathrm{mV})$. As shown in Figure $4 A$, the $\mathrm{N}$-type current again plays a major role in activating the 
mAHP, because the application of $300 \mathrm{~nm}$ cono-GV IA reduced it drastically. A summary of the results obtained with separate applications of the various antagonists is shown in Figure $4 B$. Cono-GVIA (200-400 nM) was the dominant blocker, inhibiting $58.7 \pm 15.7 \%$ of the mAHP, nifedipine $(3 \mu \mathrm{M})$ inhibited $24.1 \pm$ $5.8 \%$ of this AHP, and Aga-IVA (100 nM) blocked $10.0 \pm 3.8 \%$ of it, whereas Aga-IVA at 300-500 nM (added after $100 \mathrm{~nm}$ Aga-IVA) blocked only $7.4 \pm 3.7 \%$ of the mAHP. Comparable inhibitory effects on the mAHP were observed during cumulative application of the HVA antagonists. Indeed, nifedipine $(3 \mu \mathrm{M})$ reduced the mAHP by $20.2 \pm 6.9 \%(n=5)$; adding cono-GVIA $(200-400 \mathrm{nM})$ reduced it by $48.4 \pm 6.9 \%(n=5)$, whereas further addition of Aga-IVA (100 nM) produced no further inhibition.

Amiloride was used next in an attempt to assess the role of T-type channels in the burst-evoked mAHP (Matthews and Lee, 1991; Gorelova and Reiner, 1996). However, because it completely blocked burst firing (Fig. 4C,D) and converted it into tonic firing, amiloride could not be used for this purpose. Therefore, the effects of amiloride, and hence the implication of T-type channels, were investigated in the mAHP evoked by one AP. Single APs were elicited from a membrane potential of $-60 \mathrm{mV}$ with 1-msec-long depolarizing current steps. Figure $4 E$ shows in control conditions an AP followed by a depolarizing afterpotential (DAP, open arrowhead) and a consecutive mAHP (arrow), which is reduced by approximately one-half in the presence of $300 \mu \mathrm{M}$ amiloride. In addition, it is noteworthy that amiloride $(n=6)$ or cadmium $(n=2)$ completely blocked the DAP, indicating that this depolarization is linked to the activation of a T-type current. Cumulative application of $400 \mathrm{~nm}$ cono-GVIA abolished the remaining mAHP (Fig. 4E). Altogether (Fig. 4F), $300 \mu \mathrm{M}$ amiloride reduced the mAHP by $34.1 \pm 9.9 \%$ and $3 \mu \mathrm{M}$ nifedipine had no effect, whereas cono-GVIA inhibited $65.9 \pm$ $9.9 \%$ of the remaining AHP. Because no effect of nifedipine was found after amiloride application, the effect of nifedipine on the mAHP after a burst (Fig. 3B) thus seems to result from a nonspecific blocking effect of nifedipine on T-type channels. Taken together, these data indicate that calcium entering through $\mathrm{N}$ - and T-type channels is mainly responsible for activating the apamin-sensitive mAHP.

\section{DISCUSSION}

The major goal of the present study was to examine the relative contribution of the different calcium current types to the genesis of AHPs in cholinergic NB neurons. After the demonstration that these cells possess at least five types of pharmacologically distinguishable HVA currents as well as an amiloride-sensitive T-type current, we demonstrated that the dominant current contributing to their AHPs is the N-type current although, depending on their different firing patterns (and different associated AHPs), either Por T-type currents could be involved also.

\section{Identification of neurons}

Within the NB, cholinergic and GABAergic neurons have been shown to coexist and together comprise the majority of the large neurons (Gritti et al., 1993). It is shown here that dissociated NB neurons also can be classified into two groups on the basis of the size of their soma. Indeed, neurons $>25 \mu \mathrm{M}$ were mostly cholinergic $(\sim 80 \%)$, whereas those that were $<25 \mu \mathrm{M}$ comprised both ChAT-positive and ChAT-negative neurons. These results indicate that, in this preparation, a criterion of size represents a reasonable method to distinguish cholinergic from noncholinergic neurons, as was proposed initially by Nakajima et al. (1985).
The electrophysiological properties of the larger neurons in this study confirmed this contention, because they corresponded closely to those of immunohistochemically identified cholinergic NB neurons in basal forebrain slices (Khateb et al., 1992; Alonso et al., 1994, 1996). Indeed, in whole-cell current-clamp recordings, almost all neurons of $>25 \mu \mathrm{M}$ in diameter displayed LTS activity, permitting them to discharge in bursts of two to three spikes when they were stimulated from a hyperpolarized membrane potential.

\section{NB cholinergic neurons possess at least five types of HVA calcium channels}

It has been demonstrated for a wide variety of cell types that the whole-cell HVA calcium current can be separated into several subtypes by using different calcium channel antagonists (Mintz et al., 1992; Randall and Tsien, 1995; McDonough et al., 1996). In cholinergic NB neurons the total HVA current was composed of $\sim 17 \%$ L-type, 35\% N-type, 10\% P-type, 20\% Q-type, and 18\% R-type. Although both L- and N-type currents were characterized before in cholinergic basal forebrain neurons (Allen et al., 1993), P-, Q-, and R-types had not been described before. The demonstration of the presence of both P- and Q-type currents in these neurons relies on the observation that low and high concentrations of Aga-IVA block distinct channels (Sather et al., 1993; Randall and Tsien, 1995; Ciranna et al., 1996). Although an important number of studies has revealed that $100 \mathrm{~nm}$ Aga-IVA is saturating for the P-type channels in many preparations (Mintz et al., 1992; Mintz and Bean, 1993), more recent studies have shown that these channels actually could be blocked by low concentrations of Aga-IVA ( IC $_{50}$ of 1-2 nM; Randall and Tsien, 1995; Ciranna et al., 1996). These latter studies have shown, in addition, that other channels, presumably Q-type channels, could be inhibited ( $\mathrm{IC}_{50}$ of $\sim 90-200 \mathrm{nM}$ ) with higher concentrations of Aga-IVA. Another indication for the distinction between a Pand a Q-type current is based on the different time to peak of the components sensitive to low and high Aga-IVA concentrations. Indeed, similar to other studies that have indicated a faster time to peak for P-type than for Q-type currents (Mintz et al., 1992; Sather et al., 1993; Randall and Tsien, 1995), the present study revealed a faster time to peak of the component sensitive to a lower concentration of Aga-IVA. In addition, we observed a component that was resistant to all HVA antagonists as well as to amiloride but that was blocked by cadmium. This calcium current peaked at $0 \mathrm{mV}$, a value more positive than that of the R-type current in cerebellar neurons (Randall and Tsien, 1995) and that of the current flowing through $\alpha 1 \mathrm{E}$ subunits expressed in oocytes (Ellinor et al., 1993). Notwithstanding this discrepancy, the usual terminology of R-type current was used for the resistant component of the HVA current in cholinergic NB neurons.

Finally, it is worth mentioning that activation and deactivation kinetics of HVA calcium currents may lead to different contributions of calcium current subtypes during a $40 \mathrm{msec}$ voltage step and during a brief AP. This issue has been addressed previously in sympathetic neurons (Toth and Miller, 1995). In that preparation, APs are long-lasting, having a half-width of $\sim 10 \mathrm{msec}$, making AP waveforms a possible tool to voltage-clamp currents. However, APs of cholinergic NB neurons are relatively short $(<2$ msec at half-width), making a similar strategy more difficult to use for quantitative assessment of currents in these cells.

\section{Differential contributions of calcium channels to AHPs}

In cholinergic NB neurons the N-type current was the one principally involved in the SAHP. Indeed, although only $35 \%$ of the 
total HVA current consisted of calcium flowing through N-type channels, $\sim 60 \%$ of this AHP was blocked by cono-GVIA, the selective N-type channel blocker. Similarly, although only $\sim 10 \%$ of the calcium current was considered to be of the P-type, $\sim 20 \%$ of the sAHP was activated by calcium flowing through P-type channels. In contrast, current types $\mathrm{L}$ and $\mathrm{Q}$ contributed little to the SAHP. Also noteworthy was the lack of effect of amiloride on the sAHP. During the mAHP (elicited by one or a burst of APs) the N-type current was again dominant, but the P-type current played a minor role. On the other hand, contrary to the SAHP, the T-type current was also important in the activation of the mAHP. To our knowledge this represents the first demonstration that a LVA calcium current is involved in the genesis of the mAHP. The fact that the T-type current participates in the activation of AHPs provides an important mechanism that would favor the deinactivation of the LVA current and thus promote regenerative oscillatory events. The L-type channel blocker nifedipine also produced a significant reduction of the mAHP elicited by a burst. However, this reduction may be partly attributable to a direct block of the LVA current by nifedipine (Allen et al., 1993; present study) because the addition of nifedipine after that of amiloride did not have a significant effect on the mAHP. Dihydropyridines such as nifedipine and nimodipine have been shown previously in a variety of cell types (including basal forebrain cholinergic neurons) to have nonspecific effects on T-type channels in addition to their well known antagonization of L-type channels (Akaike, 1991; Allen et al., 1993).

In cholinergic NB neurons the mAHP is mediated by smallconductance apamin-sensitive $\mathrm{K}_{\mathrm{Ca}}$ channels, whereas the sAHP is mediated by the recently described small-conductance apaminresistant $\mathrm{K}_{\mathrm{Ca}}$ channels (Lancaster et al., 1991; Kohler et al., 1996; Sah, 1996). We therefore speculate that apamin-sensitive $K_{C a}$ channels preferentially interact with $\mathrm{N}$ - and T-type calcium channels, whereas apamin-resistant $\mathrm{K}_{\mathrm{Ca}}$ channels interact with $\mathrm{N}$-type and, to a lesser extent, P-type channels. This preferential interaction may be the result of a special colocalization between potassium and calcium channel subtypes and/or characteristics proper to the calcium channels themselves (including in particular their activation and inactivation kinetics). In addition, the kinetics of calcium diffusion and reuptake in the cell might play a role in this preferential interaction. There is earlier evidence in the literature for preferential colocalization between voltageactivated calcium channels and $\mathrm{K}_{\mathrm{Ca}}$ channels. At the motoneuron terminal N-type channels are located spatially close to largeconductance $\mathrm{K}_{\mathrm{Ca}}$ channels (Robitaille et al., 1993). In ciliary ganglion cells L-type, but not N-type, channels specifically can activate large-conductance $\mathrm{K}_{\mathrm{Ca}}$ (Wisgirda and Dryer, 1994). In other cell types $\mathrm{N}$-type channels can activate apamin-sensitive $\mathrm{K}_{\mathrm{Ca}}$ channels, generating the AHP after a single spike (Viana et al., 1993; Umemiya and Berger, 1994; Gorelova and Reiner, 1996). Finally, in vagal neurons (Sah, 1995) L- and N-type calcium currents have been shown to contribute to the apamin-sensitive mAHP, but not to the large-conductance charybdotoxin-sensitive AHP. However, to our knowledge a detailed analysis of the precise contribution of all the different calcium channel subtypes to both mAHP and sAHP in the same type of neurons has not been attempted before.

\section{Physiological significance}

Although the exact role of this preferential interaction between voltage-gated calcium channel subtypes and $\mathrm{K}_{\mathrm{Ca}}$ channels is unknown, this phenomenon may allow neurons to optimize the inhibitory mechanism induced by the AHP. In cholinergic NB neurons one could speculate that during tonic slow single-spike firing and short rhythmic bursting, calcium entering through $\mathrm{T}$ and N-type channels would play a pivotal role in the activation of the apamin-sensitive AHP, thereby contributing to regenerative membrane oscillations. However, during prolonged activations an additional (apamin-insensitive) $\mathrm{K}_{\mathrm{Ca}}$ current could be recruited that would be activated essentially through $\mathrm{N}$ - and P-type channels.

Finally, it is of interest to note that, the delayed AHPs in cholinergic neurons being calcium-dependent, the rhythmicity that these cells can adopt (in conjunction with the LTS) differs from that of thalamic relay cells (Jahnsen and Llinás, 1984; Steriade and Llinás, 1988; McCormick, 1992), which rather depends on a cesium-sensitive $I_{\mathrm{h}}$ (McCormick and Pape, 1990a). Accordingly, in these two neuronal systems implicated in the control of behavioral states, the modulation by afferents ascending from pontomesencephalic neurons also differs. Indeed, whereas the $I_{\mathrm{h}}$ is an important site of action of brainstem transmitters on thalamic cells (McCormick and Pape, 1990b; McCormick, 1992), it is the calcium-dependent AHP (by an effect on calcium currents) that often is targeted in NB cholinergic neurons (A. Khateb, unpublished data; Williams et al., 1997).

\section{REFERENCES}

Akaike N (1991) T-type calcium channel in mammalian CNS neurones. Comp Biochem Physiol [C] 98:31-40.

Allen TG, Sim JA, Brown DA (1993) The whole-cell calcium current in acutely dissociated magnocellular cholinergic basal forebrain neurones of the rat. J Physiol (Lond) 460:91-116.

Alonso A, Faure MP, Beaudet A (1994) Neurotensin promotes oscillatory bursting behavior and is internalized in basal forebrain cholinergic neurons. J Neurosci 14:5778-5792.

Alonso A, Khateb A, Fort P, Jones BE, Mühlethaler M (1996) Differential oscillatory properties of cholinergic and non-cholinergic nucleus basalis neurons in guinea pig brain slice. Eur J Neurosci 8:169-182.

Bertrand D, Buisson B, Krause RM, Bertrand S (1997) Electrophysiology: a method to investigate the functional properties of ligand-gated channels. J Recept Signal Transduct Res 17:227-242.

Ciranna L, Feltz P, Schlichter R (1996) Selective inhibition of highvoltage-activated $\mathrm{L}$-type and Q-type $\mathrm{Ca}^{2+}$ currents by serotonin in rat melanotrophs. J Physiol (Lond) 490:595-609.

Ellinor PT, Zhang J-F, Randall AD, Zhou M, Schwartz TL, Tsien RW, Horne WA (1993) Functional expression of a rapidly inactivating neuronal calcium channel. Nature 363:455-458.

Gorelova N, Reiner PB (1996) Role of the afterhyperpolarization in control of discharge properties of septal cholinergic neurons in vitro. J Neurophysiol 75:695-706.

Griffith WH, Taylor L, Davis MJ (1994) Whole-cell and single-channel currents in guinea-pig basal forebrain neurons. J Neurophysiol 71:2359-2376.

Gritti I, Mainville L, Jones BE (1993) Codistribution of GABA- with acetylcholine-synthesizing neurons in the basal forebrain of the rat. J Comp Neurol 329:438-457.

Hamill OP, Marty A, Neher E, Sakmann B, Sigworth FJ (1981) Improved patch-clamp techniques for high-resolution current recording from cells and cell-free membrane patches. Pflügers Arch 391:85-100.

Jahnsen H, Llinás R (1984) Ionic basis for the electroresponsiveness and oscillatory properties of guinea-pig thalamic neurones in vitro. J Physiol (Lond) 349:227-247.

Jones BE (1993) The organization of central cholinergic systems and their functional importance in sleep-waking states. Prog Brain Res 98:61-67.

Jones BE (1994) Reticular formation. Cytoarchitecture, transmitters and projections. In: The nervous system of the rat (Paxinos G, ed), pp 155-171. New South Wales, Australia: Academic.

Kay AR, Wong RKS (1986) Isolation of neurons suitable for patchclamping from adult mammalian central nervous systems. J Neurosci Methods 16:227-238.

Khateb A, Mühlethaler M, Alonso A, Serafin M, Jones BE (1992) 
Cholinergic nucleus basalis neurons display the capacity for rhythmic bursting activity mediated by low-threshold calcium spikes. Neuroscience 51:489-494.

Khateb A, Fort P, Alonso A, Jones BE, Mühlethaler M (1993) Pharmacological and immunohistochemical evidence for serotonergic modulation of cholinergic nucleus basalis neurons. Eur J Neurosci 5:541-547.

Khateb A, Fort P, Serafin M, Jones BE, Mühlethaler M (1995) Rhythmical bursts induced by NMDA in guinea-pig cholinergic nucleus basalis neurones in vitro. J Physiol (Lond) 487:623-638.

Kohler M, Hirschberg B, Bond CT, Kinzie JM, Marrion NV, Maylie J, Adelman JP (1996) Small-conductance, calcium-activated potassium channels from mammalian brain. Science 273:1709-1714.

Lancaster B, Nicoll RA, Perkel DJ (1991) Calcium activates two types of potassium channels in rat hippocampal neurons in culture. J Neurosci 11:23-30.

Markram H, Segal M (1990) Electrophysiological characteristics of cholinergic and non-cholinergic neurons in the rat medial septum-diagonal band complex. Brain Res 513:171-174.

Matthews RT, Lee WL (1991) A comparison of extracellular and intracellular recordings from medial septum/diagonal band neurons in vitro. Neuroscience 42:451-462.

McCormick DA (1992) Neurotransmitter actions in the thalamus and cerebral cortex and their role in neuromodulation of thalamocortical activity. Prog Neurobiol 39:337-388.

McCormick DA, Pape H-C (1990a) Properties of a hyperpolarizationactivated cation current and its role in rhythmic oscillations in thalamic relay neurones. J Physiol (Lond) 431:291-318.

McCormick DA, Pape H-C (1990b) Noradrenergic and serotonergic modulation of a hyperpolarization-activated cation current in thalamic relay neurones. J Physiol (Lond) 431:319-342.

McDonough SI, Swartz KJ, Mintz IM, Boland L, Bean BP (1996) Inhibition of calcium channels in rat central and peripheral neurons by $\omega$-conotoxin MVIIC. J Neurosci 16:2612-2623.

Mesulam MM (1995) Structure and function of cholinergic pathways in the cerebral cortex, limbic system, basal ganglia, and thalamus of the human brain. In: Psychopharmacology: the fourth generation of progress (Bloom FE, Kupfer DJ, eds), pp 135-146. New York: Raven.

Mintz M, Bean BP (1993) GABA B $_{\text {r }}$ receptor inhibition of P-type $\mathrm{Ca}^{2+}$ channels in central neurons. Neuron 10:889-898.

Mintz M, Adams ME, Bean BP (1992) P-type calcium channels in rat central and peripheral neurons. Neuron 9:85-95.

Murchison D, Griffith WH (1996) High-voltage-activated calcium currents in basal forebrain neurons during aging. J Neurophysiol 76:158-174.

Nakajima Y, Nakajima S, Obata K, Carlson GC, Yamaguchi K (1985) Dissociated cell culture of cholinergic neurons from nucleus basalis of Meynert and other basal forebrain nuclei. Proc Natl Acad Sci USA 82:6325-6329.

Nuñez A (1996) Unit activity of rat basal forebrain neurons: relationship to cortical activity. Neuroscience 72:757-766.

Paxinos G, Watson C (1986) The rat brain in stereotaxic coordinates. Sydney: Academic.
Penington NJ, Kelly JS (1993) Ionic dependence of a slow inward tail current in rat dorsal raphe neurones. J Physiol (Lond) 464:33-48.

Randall A, Tsien RW (1995) Pharmacological dissection of multiple types of $\mathrm{Ca}^{2+}$ channel currents in rat cerebellar granule neurons. J Neurosci 15:2995-3012.

Robitaille R, Garcia ML, Kaczorowski GJ, Charlton MP (1993) Functional colocalization of calcium and calcium-gated potassium channels in control of transmitter release. Neuron 11:645-655.

Sah P (1995) Different calcium channels are coupled to potassium channels with distinct physiological roles in vagal neurons. Proc R Soc Lond [Biol] 260:105-111.

Sah P (1996) $\mathrm{Ca}^{2+}$-activated $\mathrm{K}^{+}$currents in neurones: types, physiological roles, and modulation. Trends Neurosci 19:150-154.

Sather WA, Tanabe T, Zhang J-F, Mori Y, Adams ME, Tsien RW (1993) Distinctive biophysical and pharmacological properties of Class A (B1) calcium channels $\alpha 1$ subunits. Neuron 11:291-303.

Stea A, Tomlinson WJ, Soong TW, Bourinet E, Dubel SJ, Vincent SR, Snutch TP (1994) The localization and functional properties of a rat brain $\alpha 1 \mathrm{~A}$ calcium channel reflect similarities to neuronal Q- and P-type channels. Proc Natl Acad Sci USA 91:10576-10580.

Steriade M, Llinás RR (1988) The functional states of the thalamus and the associated neuronal interplay. Physiol Rev 68:649-742.

Tang C-M, Presser F, Morad M (1988) Amiloride selectively blocks the low-threshold (T) calcium channel. Science 240:213-215.

Tatsumi H, Katayama Y (1993) Regulation of the intracellular free calcium concentration in acutely dissociated neurones from rat nucleus basalis. J Physiol (Lond) 464:165-181.

Tatsumi H, Katayama Y (1995) $\mathrm{Na}^{+}$-dependent $\mathrm{Ca}^{2+}$ influx induced by depolarization in neurons dissociated from rat nucleus basalis. Neurosci Lett 196:9-12.

Toth TP, Miller RJ (1995) Calcium and sodium currents evoked by action potential waveform in rat sympathetic neurones. J Physiol (Lond) 485:43-57.

Umemiya M, Berger AJ (1994) Properties and function of low- and high-voltage-activated $\mathrm{Ca}^{2+}$ channels in hypoglossal motoneurons. J Neurosci 14:5652-5660.

Viana F, Bayliss DA, Berger AJ (1993) The role of multiple potassium conductances in action potential repolarization and repetitive firing behavior of neonatal rat hypoglossal motoneurons. J Neurophysiol 69:2150-2163.

Williams S, Serafin M, Mühlethaler M, Bernheim L (1997) Facilitation of N-type calcium current is dependent on the frequency of action potential-like depolarizations in dissociated cholinergic basal forebrain neurons of the guinea pig. J Neurosci 17:1625-1632.

Wisgirda ME, Dryer SE (1994) Functional dependence of $\mathrm{Ca}^{2+}$. activated $\mathrm{K}^{+}$current on $\mathrm{L}$ - and $\mathrm{N}$-type $\mathrm{Ca}^{2+}$ channels: differences between chicken sympathetic and parasympathetic neurons suggest different regulatory mechanisms. Proc Natl Acad Sci USA 91:2858-2862.

Zhang L, Weiner JL, Valiente TA, Velumian AA, Watson PL, Jahromi SS, Schertzer S, Pennefather P, Carlen P (1994) Whole-cell recording of the $\mathrm{Ca}^{2+}$-dependent slow afterhyperpolarization in hippocampal neurones: effects of internally applied anions. Pflügers Arch 426:247-253. 